\title{
The diagnostic accuracy of diffusion weighted magnetic resonance (MR) imaging for discrimination of malignant from benign cervical lymphadenopathy in head and neck tumours using histopathology as the reference standard
}

\author{
Mathur M. ${ }^{1}$, Duhan V. ${ }^{2}$, Gupta R.K. ${ }^{3}$, Gupta S. ${ }^{4}$, Kaur N. ${ }^{5}$, Mathur A. ${ }^{6}$ \\ ${ }^{1}$ Dr. Manoj Mathur, Associate Professor, ${ }^{2}$ Dr. Vikas Duhan, Junior Resident, Department of Radio-Diagnosis, \\ Rajindra Hospital, G.M.C. Patiala, ${ }^{3}$ Dr. Rajiv Kamal Gupta, Associate Professor, Department of Pathology, \\ Rajindra Hospital, G.M.C. Patiala, ${ }^{4}$ Dr. Saryu Gupta, Associate Professor, ${ }^{5}$ Dr. Navkiran Kaur, Professor and \\ Head, ${ }^{6}$ Dr. Aditi Mathur, Intern, Department of Radio-Diagnosis, Rajindra Hospital, G.M.C. Patiala, Punjab, \\ India.
}

Address for Correspondence: Dr. Vikas Duhan, Junior Resident, Department of Radio-Diagnosis, Rajindra Hospital, G.M.C. Patiala. Email: duhanv7@gmail.com.

\begin{abstract}
Head and neck cancers accounts for maximum number of cancer cases in Indian hospital settings. Involvement of neck nodes is a very important prognostic factor of its outcome. Differentiation between benign and metastatic lymphadenopathy often presents a diagnostic challenge with conventional imaging techniques. Diffusion-weighted imaging (DWI) has emerged as a powerful non-invasive imaging technique that is capable of characterisation of these lesions as benign or malignant with the help of apparent diffusion coefficient (ADC) maps. Aim: The aim of this cross-sectional study was to determine the diagnostic accuracy of DWI to differentiate benign from malignant cervical lymph nodes in head and neck tumours, subsequently confirming the results using histopathology as the reference standard. Materials and Methods: The cross-sectional study was conducted on 60 patients of either age or sex with enlarged neck lymph nodes over a period of 2 years, subsequently these patients underwent DW MRI imaging followed by histopathology of either neck dissection specimen or core biopsy or US guided FNAC as a part of the study. Results: Out of 60 patients, 41(68.33 percent) cases came out as malignant and 19 (31.67 percent) cases came out as benign. The results obtained were 36 true positives, 4 false positives, 15 true negatives and 5 false negatives. The overall sensitivity of DWI for differentiating malignant from benign cervical lymphadenopathy was $87.80 \%$ with specificity of $78.95 \%$. The positive predictive value and negative predictive value were $90.00 \%$ and $75.00 \%$ respectively. The best ADC threshold value for distinguishing benign and malignant nodes was $1.005 \times 10^{-3} \mathrm{~mm}^{2} / \mathrm{sec}$. Conclusion: DWI is an important tool to differentiate benign vs malignant lymphadenopathy and helps in guiding the clinician to treat these nodes accordingly.
\end{abstract}

Keywords: Diffusion Weighted Imaging (DWI), cervical lymphadenopathy in head and neck tumours, ADC values, Histopathology reference standard.

\section{Introduction}

Head and cancer represents one of the most common cancers worldwide. More than $90 \%$ of head and neck cancers are squamous cell carcinomas (HNSCC) that arise from the mucosal

Manuscript received: $8^{\text {th }}$ December 2017

Reviewed: $18^{\text {th }}$ December 2017

Author Corrected: $26^{\text {th }}$ December 2017

Accepted for Publication: $31^{\text {st }}$ December 2017 surfaces of the oral cavity [1]. Lymphatic spread to regional lymph nodes is seen early in the course of disease [2,3]. The Lymph nodal tumour spread is the most important prognostic factor in Head and neck tumours. Thus, there is an increasing need for a non-invasive technique to differentiate benign from malignant cervical lymphadenopathy. Various 


\section{Original Research Article}

modalities that can be used for this purpose are ultrasound (US), computed tomography (CT) and conventional magnetic resonance imaging (MRI). Although localization and characterization of Head and neck lesions have been well defined by $\mathrm{CT}$ and MRI, these conventional methods perform poorly in assessment of lymph node metastasis [4,5]. SPECT (single photon emission CT) and PET (photon Emission tomography) are new imaging techniques which supply functional information, [68] but they are expensive, not available in all health care centers and provides low spatial resolution.

DW imaging is a MR technique that depicts molecular diffusion, which is the brownian motion of water protons in biologic tissues [9]. It can be used to calculate ADC values of tissues in the region of interest [10]. As different type of tissues shows different $\mathrm{ADC}$ values, this variation in $\mathrm{ADC}$ values can be used as some important diagnostic criteria for differentiation of benign from malignant cervical lymphadenopathy $[10,11]$.

The aim of this cross-sectional study was to define the diagnostic accuracy of DWI to differentiate benign from malignant cervical lymph nodes.

\section{Material and Methods}

This study was performed in The Department of Radiodiagnosis, Rajindra Hospital, G.M.C. Patiala. In this cross-sectional study, a total of sixty patients of either age or sex with enlarged neck lymph nodes were considered over a period of 2 years, subsequently these patients underwent DW MRI imaging followed by histopathology of either neck dissection specimen or core biopsy or US guided Fine needle aspiration cytology (FNAC) as a part of the study. Institutional approval for conducting the study was obtained. Informed consent was taken from all the subjects before starting the study.

\section{Inclusion criteria}

1. Patients with enlarged neck nodes.

2. Patients giving consent for MRI imaging and histopathology examination (Either by core biopsy or neck dissection or FNAC)

\section{Exclusion criteria}

1. Patients with metallic implants.

2. Claustrophobic patients.

3. Patients not giving consent
The permission for conducting the study was obtained from institutional review board.

M.R. Imaging Technique- All the studies were performed by 1.5-T superconductive scanner (Siemens 1.5T Magnetom Aera MRI machine). A standard receive-only head and neck coil was used for both conventional imaging and diffusionweighted MR imaging to include nodes from the base of the skull to the suprasternal notch. We first performed routine conventional MRI sequences followed by diffusion- weighted imaging of these patients. After localiser images in axial, coronal and sagittal planes, conventional images including T1-weighted images $(600 / 15$ repetition time ms/echo time ms) and T2-weighted turbo spin echo images (4000/100) were obtained in the axial and coronal planes, with a section thickness of 3-4 mm, an interslice gap of $1 \mathrm{~mm}$, a field of view (FOV) of $240 \mathrm{~mm}$ and a flip angle of $150^{\circ}$. Diffusionweighted images were obtained in the axial plane with $5 \mathrm{~mm}$ slice thickness, $1 \mathrm{~mm}$ interslice gap, FOV $250 \mathrm{~mm}$, repetition time/echo time 5500 $\mathrm{ms} / 70 \mathrm{~ms}$.

The diffusion sensitising gradient was applied in all three orthogonal planes $(\mathrm{X}, \mathrm{Y}, \mathrm{Z})$ using $\mathrm{b}$ values $(0$ and $800 \mathrm{~mm}^{2} / \mathrm{sec}$.). The ADC map was automatically reconstructed by a standard software imager in the main console. The ADC was measured by manually placing regions of interest on the ADC map.

MRI Interpretation- The lymph nodes were characterised on the basis of internationally accepted standards for evaluating anatomic imaging data. First, the morphological features of each lymph node of interest were recorded.

Lymph nodes were considered suspicious for metastasis if they showed one of these morphological characteristic-

a) Lymph node with minimal axial diameter $>1.5 \mathrm{~cm}$.

b) Round shape of lymph nodes.

c) Any size or shape with spiculated or irregular margins.

d) Heterogenous signal intensity on T2 weighted images.

Secondly, the DW images and their corresponding ADC maps were analyzed in consensus at a picture 


\section{Original Research Article}

archiving and communication system workstation. The lymph nodes were localized on the images obtained with a b value of $0 \mathrm{~mm}^{2} / \mathrm{sec}$. For quantitative assessment, regions of interest were placed in the lymph nodes identified on the b 0 images, and the software automatically copied these regions onto the other $b$ value images. ADC maps generated from DWI were used for the measurement of ADC values.

The nodal ADC value was obtained by drawing a region of interest (ROI) covering the solid portions of the pathologic nodes in the sections in which it was present and averaging the results. In this study, we excluded the necrotic areas from analysis to avoid a false high ADC values due to the low amount of intact tumoral cells.
The results of the measurements on MRI were compared with the results of the histopathology examination which were used as the reference standard.

Statistical analysis- Data were statistically described in terms of mean \pm standard deviation $( \pm \mathrm{SD})$, and range, or frequencies (number of cases) and percentages when appropriate. For comparing categorical data, Chi square $\left(\chi^{2}\right)$ test was performed. Sensitivity, specificity, positive predictive value (PPV) and negative predictive value (NPV) were calculated. Positive Differences in ADC findings between benign and malignant lymphadenopathy were calculated on the basis of P-value using SPSS 16 version.

\section{Results}

A Total of 60 patients were included in the study. Out of 60 patients, 41(68.33 percent) cases were malignant and 19 (31.67 percent) cases were benign. 24 patients with metastatic lymphadenopathy included in the present study were males with commonest age distribution of 41-55 years.

Size was found to be some statistically significant criteria in our study with larger size comprising of more number of malignant cases. About 48 nodes were $1.5 \mathrm{~cm}$ or more in diameter; 31 malignant and 17 benign Lymph nodes while 12 nodes were less than $1.5 \mathrm{~cm}$ in diameter; 10 malignant and 2 benign LNs. In our study 18(30 percent) cases with round shape and 10 (16.66 percent) cases with lobulated shape came out to be malignant. While 14(23.34 percent) cases with oval shape came out as benign lymphadenopathy.

Irregular margins were seen in 16 (26.66 percent) patients with malignant lymphadenopathy and none with benign lymphadenopathy. This feature was found to be statistically significant with a $p$ value of $<0.01$. Irregular margins when present was a feature of malignancy.

Among the malignant lymphadenopathy group $(n=41), 21$ nodes exhibited homogenous solid architecture $(35 \%)$ and 20 nodes exhibited heterogeneous architecture of the parenchyma $(33.33 \%)$. Among the benign lymphadenopathy group $(n=19), 11$ nodes $(18.33 \%)$ exhibited homogenous solid architecture while 8 cases $(13.33 \%$ ) showed heterogenous architecture of the parenchyma. These changes in nodal architecture were well depicted on T2-weighted images. Presence of necrosis was seen in 20(33.33 percent) patients with malignant lymphadenopathy and 8 (13.34 percent) patients with benign lymphadenopathy. However presence of necrosis was not statistically significant in our study.

Table1: DWI (B VALUE $\left.=800 \mathrm{~mm}^{2} / \mathrm{sec}\right)$.

\begin{tabular}{|c|c|c|c|c|}
\hline \multirow{2}{*}{ DWI } & \multicolumn{2}{|c|}{ Benign } & \multicolumn{2}{c|}{ Malignant } \\
\cline { 2 - 5 } & No. of Patients & Percentage & No. of Patients & Percentage \\
\hline Absent & 15 & 25.00 & 5 & 8.33 \\
\hline Present & 4 & 6.67 & 36 & 60.00 \\
\hline Total & 19 & 31.67 & 41 & 68.33 \\
\hline Chi Square & \multicolumn{3}{|c|}{26.033} \\
\hline P value & \multicolumn{3}{|c|}{$<0.001$} \\
\hline Significance & \multicolumn{3}{|c}{ Highly significant (HS) } \\
\hline
\end{tabular}




\section{Original Research Article}

Diffusion restriction was seen in 36 (60 percent) patients and not seen in 5 ( 8.33 percent) patients with malignant lymphadenopathy. Out of 19 cases of benign lymphadenopathy, 4 cases showed presence of diffusion restriction. Diffusion restriction came out as statistically highly significant predictor of malignancy with $\mathrm{p}$ value of $<0.001$ (Table1).

Table-2: Apparent diffusion coefficient (ADC) Value.

\begin{tabular}{|c|c|c|c|c|}
\hline & $\begin{array}{c}\text { Mean } \\
\left(\mathbf{x 1 0}^{-3} \mathbf{m m}^{2} / \mathbf{s e c}\right)\end{array}$ & S.D & P Value & Significance \\
\cline { 1 - 3 } Benign & 1.28 & 0.273 & $<0.001$ & HS \\
\cline { 1 - 2 } Malignant & 0.89 & 0.241 & \\
\hline
\end{tabular}

The mean ADC value for benign lymphadenopathy came out to be $1.28 \times 10^{-3} \mathrm{~mm}^{2} / \mathrm{sec}$ with a S.D. of 0.273 and $\mathrm{ADC}$ value for malignant lymphadenopathy came out to be $0.89 \times 10^{-3} \mathrm{~mm}^{2} / \mathrm{sec}$ with a S.D. of 0.241 . Above values were found to be statistically highly significant with a p value of $<0.001$. (Table 2)

In our study after radiological analysis, 38 (63.33 percent) cases comes as malignant and 22 (36.67 percent) cases were benign.

Table-3: Histopathological Diagnosis

\begin{tabular}{|c|c|c|c|c|}
\hline \multicolumn{3}{|c|}{ Histopathological Diagnosis } & Number of Patients & Percentage \\
\hline \multirow{2}{*}{\multicolumn{2}{|c|}{ Benign }} & Reactive lymphadenitis & 12 & 20.0 \\
\hline & & Chronic Granulomatous inflammation & 7 & 11.67 \\
\hline \multirow{8}{*}{ Malignant } & \multirow{3}{*}{$\begin{array}{l}\text { Squamous cell } \\
\text { carcinoma } \\
\text { (SCC) }\end{array}$} & Well-Differentiated & 11 & 18.33 \\
\hline & & Moderately-differentiated & 8 & 13.33 \\
\hline & & Poorly-Differentiated & 5 & 8.33 \\
\hline & \multirow{2}{*}{ Lymphoma } & Hodgkin lymphoma (HL) & 7 & 11.67 \\
\hline & & Non-hodgkin lymphoma (NHL) & 6 & 10.0 \\
\hline & \multirow{3}{*}{ Others } & Papillary carcinoma thyroid & 2 & 3.33 \\
\hline & & Mucoepidermoid carcinoma parotid gland & 1 & 1.67 \\
\hline & & Malignant fibrous histiocytosis (MFH) & 1 & 1.67 \\
\hline \multicolumn{3}{|r|}{ Total } & 60 & 100.0 \\
\hline
\end{tabular}

In our study, after histopathological analysis we divided the examined lymph nodes into 2 categories: malignant lymph nodes 41 cases $(68.33 \%)$ and benign lymph nodes 19 cases $(31.67 \%)$.

Benign lymphadenopathy were further subdivided as 12 cases of reactive lymphadenitis and 7 cases of chronic granulomatous inflammation.

Malignant lymphadenopathy were further subdivided as SCC (Well differentiated 11 cases, moderately differentiated 8 cases and poorly differentiated 5 cases), lymphoma (HL 7 cases and NHL 6 cases) and others (Papillary carcinoma thyroid 2 cases, Mucoepidermoid carcinoma of parotid 1 case and MFH 1 case). (Table 3 ). 
Table-4 : Statistical Analysis.

\begin{tabular}{|c|c|}
\hline Parameters & Result \\
\hline Sensitivity & $87.80 \%$ \\
\hline Specificity & $78.95 \%$ \\
\hline PPV & $90.00 \%$ \\
\hline NPV & $75.00 \%$ \\
\hline
\end{tabular}

All benign cases were considered as true negatives and all malignant cases were true positives.

Out of 60 patients, after radiological evaluation, 38(63.33 percent) cases came out as malignant and 22 (32.67 percent) cases came out as benign. The results obtained were 36 true positives, 4 false positives, 15 true negatives and 5 false negatives. The sensitivity and specificity were $87.80 \%$ and 78.95 percent respectively for predicting nodal metastasis. The positive predictive value and negative predictive values were $90.00 \%$ and $75.00 \%$, respectively. (Table 4)

Table-5: Area under curve.

\begin{tabular}{|c|c|}
\hline \multicolumn{2}{|c|}{ Area under Curve } \\
\hline Area & P value \\
\hline 0.834 & $<0.001$ \\
\hline
\end{tabular}

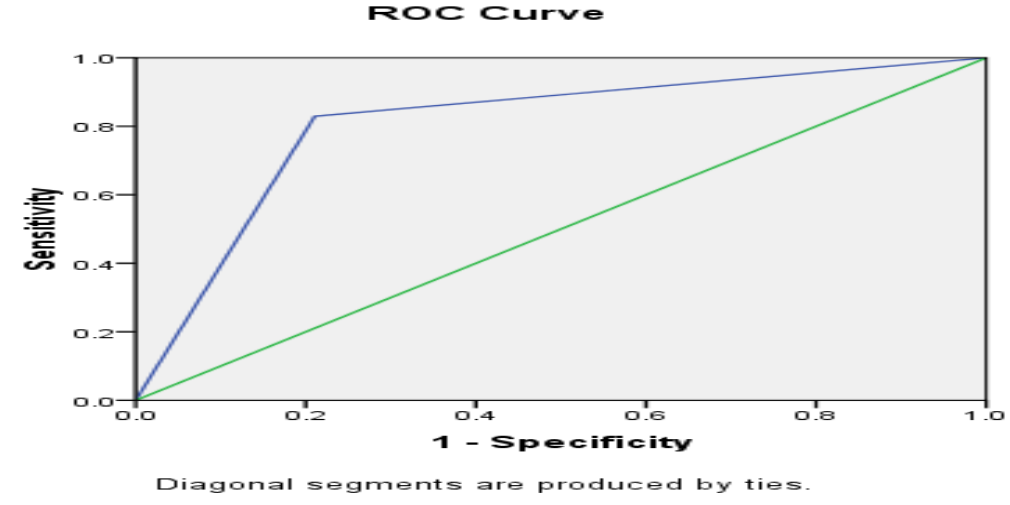

The best ADC threshold value for distinguishing benign and malignant nodes was $1.005 \times 10^{-3} \mathrm{~mm}^{2} / \mathrm{sec}$. The area under the curve was $0.834(\mathrm{p}<0.001)$ when receiver operating characteristic (ROC) curve of the ADC value was used for the differentiation of benign from malignant lymph nodes (Table 5).

\section{CASE-1}

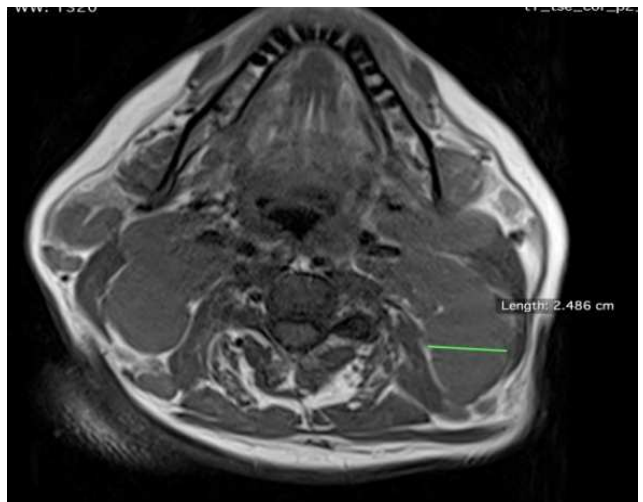

Figure (A)

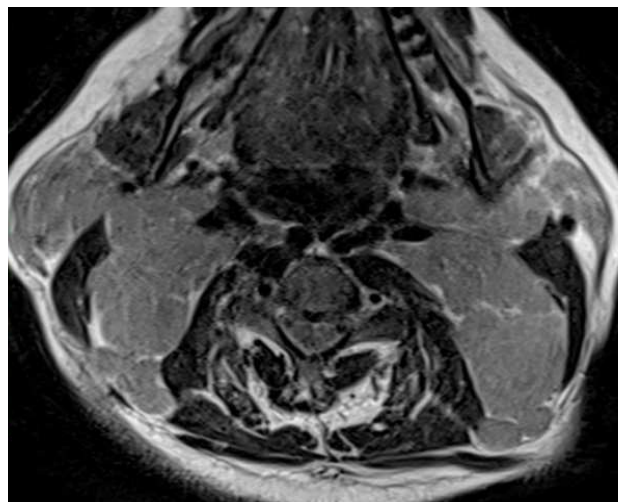

Figure (B) 


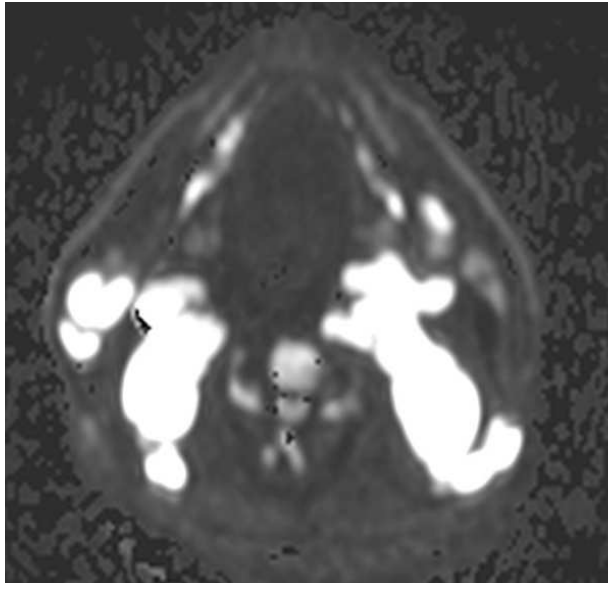

Figure (C)
Original Research Article

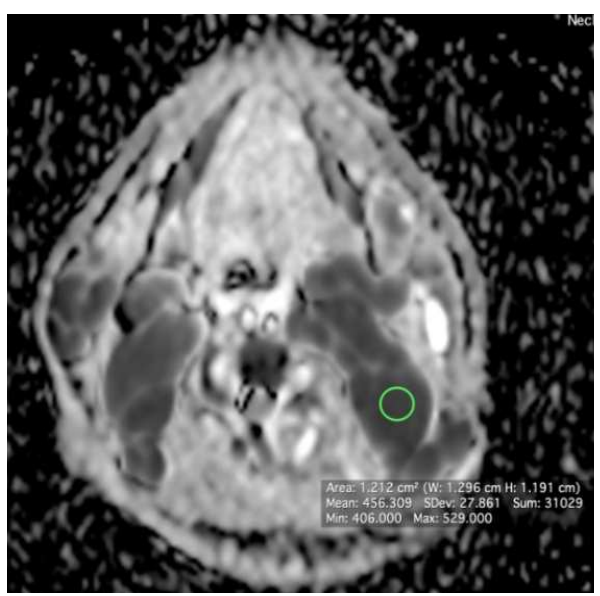

Figure (D)

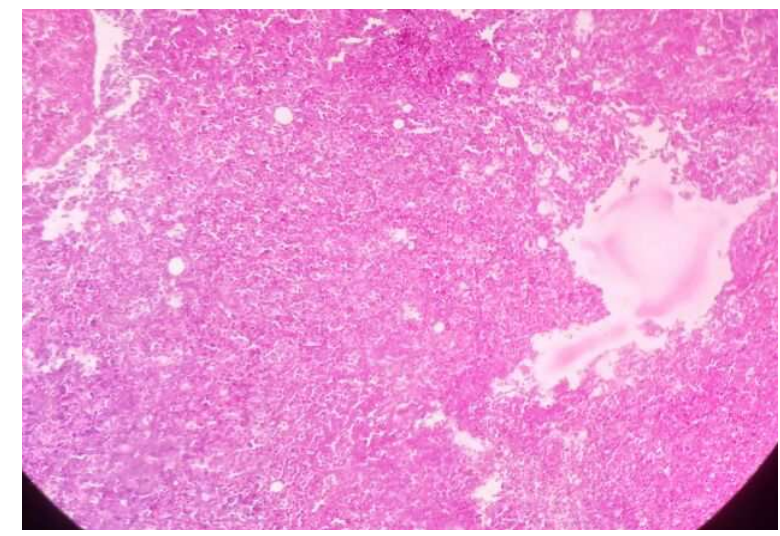

Figure (E)

A 55-year-old man with bilateral enlarged neck lymph nodes. (A) Transverse TIWI. (B) Transverse T2WI. Lymphadenopathy in bilateral IV and V levels. (C) Transverse DWI image obtained with a b factor of $800 \mathrm{~s} /$ $\mathrm{mm}^{2}$ shows high signal intensity of bilateral lymphadenopathy. (D) Transverse ADC map shows that the ADC value of the mass is $0.48 \times 10^{-3} \mathrm{~mm}^{2} / \mathrm{sec}$. (E) Histopathology shows Non Hodgkins Lymphoma.

\section{CASE-2}

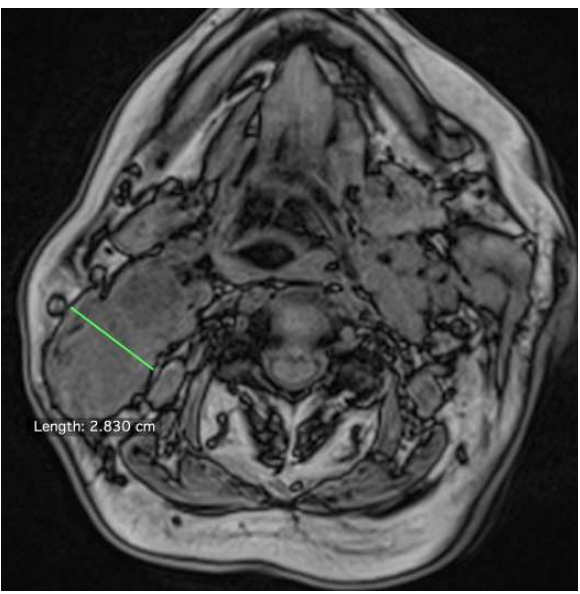

Figure (A)

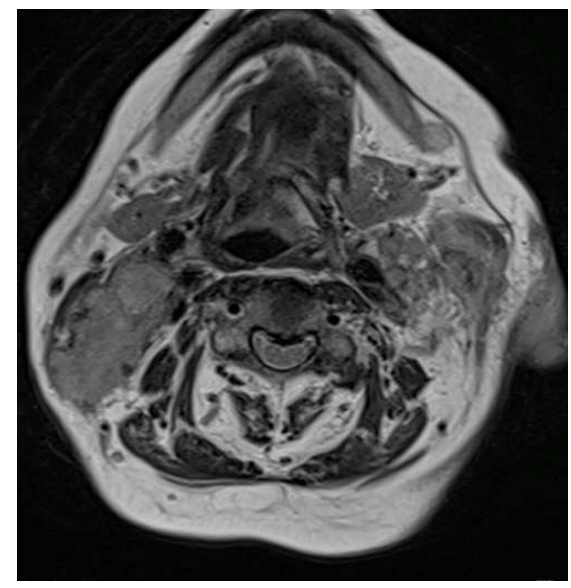

Figure (B) 


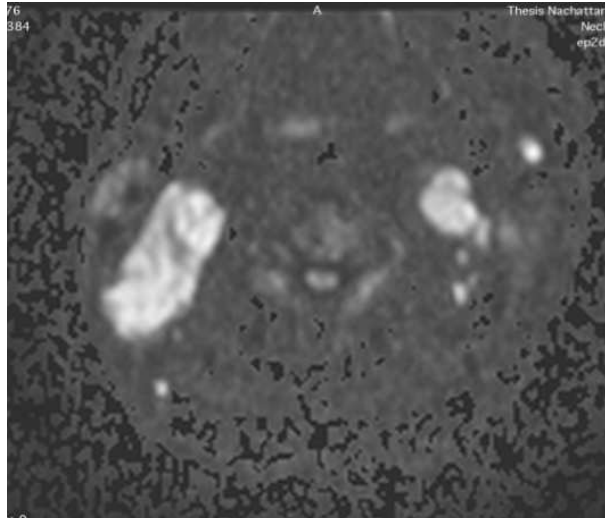

Figure (C)

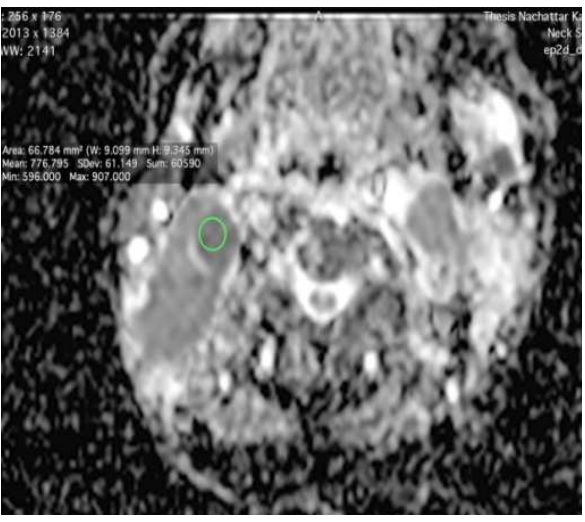

Figure (D)

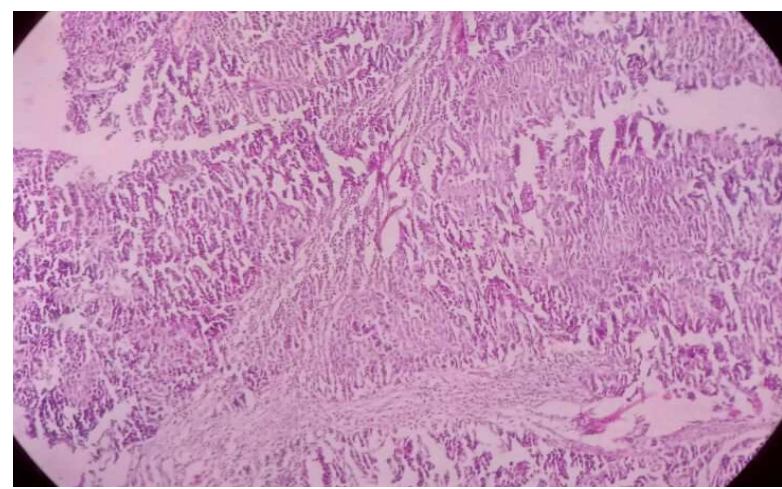

Figure (E)

A 63-year-old woman with bilateral enlarged neck lymph nodes more on right side at level II. (A) Axial TIWI showing a large isointense level II LN. (B) Axial T2WI demonstrating the LN heterogenous signal. (C) DW image at a b-value of $800 \mathrm{~s} / \mathrm{mm}^{2}$ shows enlarged hyperintense nodes at level II on both sides(more on right). (D) ADC map. Low signal of the $\mathrm{LN}$ which measured $0.77 \times 10^{-3} \mathrm{~mm}^{2} / \mathrm{sec}$ is seen, in keeping with a metastatic lymphadenopathy. (E) Histopathology reveals Poorly differentiated SCC.

\section{Discussion}

Head and neck tumours includes tumours arising from a variety of sites in the upper aero-digestive tract. Metastatic involvement of neck lymph nodes is a frequent finding for these tumour types and has a major prognostic impact on patient survival [12].

For this reason, accurate detection of nodal metastases is mandatory to optimise the treatment plan. Diffusion weighted imaging, due to its ability to probe the tumoral microstructure, its short acquisition time, its high repeatability, and safety given the absence of intravenous administration of contrast medium is the reliable noninvasive tool to differentiate between benign and metastatic lymph nodes in patients with Head and neck tumors. This cross-sectional study was conducted in The Department of Radiodiagnosis, Rajindra Hospital,
G.M.C. Patiala. It consisted of a total of sixty patients of either age or sex with enlarged neck lymph nodes and other complaints related to head and lesions over a period of 2 years. The present study was carried out to determine the diagnostic accuracy of DW MRI to differentiate benign from malignant cervical lymph nodes.

Our study had been conducted on 60 patients. The prevalence of metastatic lymphadenopathy was seen predominantly in older age groups with majority of subjects being males. Rekha et al [13] found that the highest incidence of HNSCC was seen within the age group of 51-60 $(P<0.001)$ with a male predominance pattern.

31 out of 60 patients $(56.66 \%)$ in our study with nodal size $>1.5 \mathrm{~cm}$ had positive lymph nodes. 


\section{Original Research Article}

Thus, nodal size $>1.5 \mathrm{~cm}$ favours the diagnosis of metastatic lymphadenopathy. Positive correlation was seen in the study done by Bondt et al (14). They observed a specificity of $92 \%$ for the diagnosis of metastatic lymph nodes if the size of node was more than $10 \mathrm{~mm}$.

In the present study, 10 positive nodes were also seen with lymph nodal size $<1.5 \mathrm{~cm}$ indicating that even smaller nodes can harbour metastasis. Corroborating results were shown in a study done by Berkel et al [15]. They found that metastatic lymph nodes with a minimal axial diameter smaller than $10 \mathrm{~mm}$ comprised $58 \%$ of all malignant nodes.

In the present study, amongst the metastatic lymph nodes, 19 (31.66 percent) cases had round shape and 10 (16.67 percent) cases had lobulated shape of lymph nodes, while 12 (20.00 percent) cases had oval shape. The difference was found to be statistically significant. It was determined that round shape of lymph nodes favored lymph nodal metastasis. Takes et al [16] (1998) also found round shape of lymph node as an important marker for determination of cervical nodal metastasis. Similar results were also seen in the study done by Bondt et al[14] (2009) i.e. a ratio less than 2, like in round lymph nodes versus elliptical lymph nodes, was indicative of metastatic disease.

In the present study, irregular margins were seen in 16 out of 41 malignant cases and none out of 19 benign cases of cervical lymphadenopathy. This difference showed high statistical significance $(P$ value- 0.001). It was concluded that presence of irregular margins favoured metastasis. Similar results were seen in the study done by Vandecaveye et al [5] in 2009.

They mentioned that an irregular contour was apparent in only the enlarged metastatic lymph nodes and in a limited number of enlarged benign lymph nodes. Positive correlation was also seen in a study conducted by Sumi et al [17] (2006). They concluded that nodal margins blending into the surrounding tissue were found in 58\% of metastatic nodes. Irregular margins were also found in some lymphomas $(23 \%)$ but were rarely observed in benign nodes $(9 \%)$.

In the present study, out of 41 cases of malignant lymphadenopathy, 20 cases showed heterogenous and 21 cases showed hyper intense signal intensity on T2WI. While out of 19 cases of benign lymphadenopathy, 8 cases were heterogenous and 11 cases were hyper intense on T2WI.

Heterogenous signal intensity was the morphological feature found predominantly in metastatic lymph nodes. This corroborated with the study done by Sumi et al [17] (2006) in which metastatic nodes exhibited heterogeneous architecture of the parenchyma on T2-weighted MR images. Positive correlation was also seen in the study done by de Bondt et al (14), they concluded that heterogeneous signal intensity on T2-weighted images contributed significantly to the prediction of the presence of metastatic lymph nodes.

In our study nodal necrosis was seen in 20 cases of malignant lymphadenopathy and 8 cases of benign lymphadenopathy. Positive correlation was seen in a study done by Ding et al [18] (2005). They concluded that central nodal necrosis was seen in 76 nodal levels at MR images, which were proved pathologically as metastatic. Similar results were seen by Sumi et al [19]. Central nodal necrosis was reported to occur in $32 \%$ of metastatic nodes.

Diffusion weighted imaging was done in all patients in our study. Presence or absence of diffusion restriction was noted. Mean apparent diffusion coefficient (ADC) was calculated from the solid part of the nodal lesions avoiding the necrotic areas.

Diffusion restriction was seen in 35 out of 41 malignant lymph nodes with resultant low signal on ADC maps and 7 out of 41 cases showed absence of diffusion restriction which contributed for False negative cases. This finding corroborated with the findings of study done by Jin Zhong et al [20].

They found out that on DWI, 43/48 metastatic Lymph nodes showed high signal intensity, whereas on the black and white flip images they presented as low signal. Whereas benign nodes showed low signal on DWI. Similar results were seen in the study done by Singh et al [21] in 2015. They found out that on diffusion images, 26/47 patients with malignant lymph nodes showed restricted diffusion on DWI with low signal intensity on ADC maps. 
Original Research Article

In our study 15/19 cases with inflammatory diseases, lymph nodes showed reduction of signal intensity on increasing $b$ values $(b=800)$ and intermediate signal intensity on ADC maps reflecting facilitated diffusion. 4/19 cases of benign lymphadenopathy, diagnosed as reactive lymphoid hyperplasia and chronic granulomatous infection showed increased signal on increasing $b$-values (false positive). Similar results were obtained by ElSaid et al[22] (2014). They stated that in $4 / 8$ cases with inflammatory diseases, lymph nodes showed reduction of signal intensity on increasing $\mathrm{b}$ values $(\mathrm{b}=1000$. $)$ and intermediate signal intensity on $\mathrm{ADC}$ maps reflecting facilitated diffusion. In 4/8 cases of benign lymphadenopathy, diagnosed as reactive lymphoid hyperplasia and chronic granulomatous infection showed increased signal on increasing $b$ values (false positive).

In our study the mean $\mathrm{ADC}$ value for benign lymphadenopathy was $1.28 \times 10-3 \mathrm{~mm} 2 / \mathrm{sec}$ with a S.D. of 0.273 and ADC value for malignant lymphadenopathy came out to be $0.89 x$ 10-3 $\mathrm{mm} 2 / \mathrm{sec}$ with a S.D. of 0.241 . The mean ADC value of malignant nodes was lower than the mean $\mathrm{ADC}$ value of benign nodes.

The difference between the mean $\mathrm{ADC}$ values of benign and malignant lesions was statistically significant $(P<0.0001)$. It was determined that ADC value of cervical lymph nodes is useful for differentiation of malignant from benign cervical lymph nodes. ElSaid et al [22] (2014) reported a threshold ADC value $1.005 \times 10-3 \mathrm{~mm} 2 / \mathrm{sec}$ for differentiating malignant from benign nodes.

These results were comparable to the study done by Perrone et al (23). They found out that the mean ADC value of malignant nodes was about $0.85 \times$ $10-3 \mathrm{~mm} 2 / \mathrm{sec}$, the mean value of benign nodes was $1.448 \times 10-3 \mathrm{~mm} 2 / \mathrm{sec}$; this difference was statistically significant $(p<0.01)$. The best threshold value was $1.03 \times 10-3 \mathrm{~mm} 2 / \mathrm{sec}$, obtaining a sensitivity of $100 \%$ and a specificity of $92.9 \%$. Similar findings had been illustrated by Zhong et al (20). They found out that the mean ADC value of metastatic nodes was approximately $0.849 \times 10-3 \mathrm{~mm} 2 / \mathrm{sec}$ (range: $0.738 \times 10-3$ $\mathrm{mm} 2 / \mathrm{sec}-0.960 \times 10-3 \mathrm{~mm} 2 / \mathrm{sec})$, lower than the mean value of the benign nodes $(1.443 \times 10-3$ $\mathrm{mm} 2 / \mathrm{sec}$, range: $1.037 \times 10-3 \mathrm{~mm} 2 / \mathrm{sec}-1.849 \times$ $10-3 \mathrm{~mm} 2 / \mathrm{sec})$.
In our study 4 false positive cases with low ADC values were seen, which were proved to be benign on histopathological examination. Wang et al[24] (2001) did a study which showed similar results. They found out that in benign lymph nodes, a false decrease in $\mathrm{ADC}$ may correlate with the presence of nodal reactive changes that manifest as multiple germinal centers and fibrotic stroma, which act as microstructural barriers.

In our study, after histopathological analysis we divided the examined lymph nodes into 2 categories: malignant lymph nodes 41 cases $(68.33 \%)$ and benign lymph nodes 19 cases $(31.67 \%)$. Benign lymphadenopathy were further subdivided as 12 cases of reactive lymphadenitis and 7 cases of chronic granulomatous inflammation. Malignant lymphadenopathy were further subdivided as SCC (Well differentiated 11 cases, moderately differentiated 8 cases and poorly differentiated 5 cases), lymphoma (HL 7 cases and NHL 6 cases) and others (Papillary carcinoma thyroid 2 cases, Mucoepidermoid carcinoma of parotid 1 case and MFH 1 case).

Corroborating results were seen in the study conducted by Abish et al [25] in which according to histopathological results the patients were divided into 10 patients with benign neck lymphadenopathy (lymphadenitis), 12 patients with metastasis from head and neck cancer (7 patients with well and moderately differentiated and 5 patients with poorly differentiated carcinomas) and 13 patients with nodal lymphoma (6 were nonHodgkin and 7 were Hodgkin lymphomas).

Limitations of the study: If we put $\mathrm{ADC}$ region of interest (ROI) on necrotic part of malignant lymph node, it gives high ADC value which contributes to false negative cases. T2 heterogeneity is a feature of malignant lymphadenopathy but we can see this even in benign lymphadenopathy in case of necrosis. Smaller size $(<1.5 \mathrm{~cm})$ favours the benign lymphadenopathy, however even smaller nodes are seen harbouring metastasis.

\section{Conclusion}

The Lymph nodal tumour spread is the most important prognostic factor in Head and neck tumours. The choice of work up and management highly depends on the extent of lymph node metastases. Therefore, detection of neck lymph 


\section{Original Research Article}

nodes and their differentiation into benign or malignant are important especially in patients with head and neck cancer for staging, treatment planning and follow-up of cancer. Mean apparent diffusion coefficient (ADC) was calculated from the solid part of the nodal lesions avoiding the necrotic areas. In our study the mean ADC value for benign lymphadenopathy came out to be $1.28 \mathrm{x}$ $10^{-3} \mathrm{~mm}^{2} / \mathrm{sec}$ with a S.D. of 0.273 and ADC value for malignant lymphadenopathy came out to be $0.89 \times 10^{-3} \mathrm{~mm}^{2} / \mathrm{sec}$ with a S.D. of 0.241 .

The mean $\mathrm{ADC}$ value of malignant nodes was lower than the mean $\mathrm{ADC}$ value of benign nodes. A threshold ADC value $1.005 \times 10^{-3} \mathrm{~mm}^{2} / \mathrm{sec}$ for differentiating malignant from benign nodes was used. So, $\mathrm{ADC}$ is an important tool in differentiating benign vs malignant lymphadenopathy.

\section{Funding: Nil, Conflict of interest: None Permission of IRB: Yes}

\section{References}

1. Vigneswaran $\mathrm{N}$ and Williams MD. Epidemiological Trends in Head and Neck Cancer and Aids in Diagnosis. Oral and maxillofacial surgery clinics of North America. 2014;26(2):123-141.

2. Kumaran PS, Thangaswamy SV, Navaneetham A. The need for early detection of neck nodal metastasis in squamous cell carcinoma of oral cavity. J Pharm Bioallied Sci 2012 ;4(2):S341-3.

3. Leusink FK, van Es RJ, de Bree R, Baatenburg de Jong RJ, van Hooff SR, Holstege FC, et al. Novel diagnostic modalities for assessment of the clinically node-negative neck in oral squamous-cell carcinoma. Lancet Oncol 2012Dec;13(12):e554-61.

4. Castelijns JA, van den Brekel MW. Imaging of lymphadenopathy in the neck. Eur Radiol 2002; 12:727-38.

5. Vandecaveye V, De Keyzer F, Vander Poorten V, Dirix P, Verbeken E, Nuyts S, et al. . Head and neck squamous cell carcinoma: value of diffusionweighted MR imaging for nodal staging. Radiology 2009;251:134-46.

6. Yamazaki Y, Saitoh M, Notani K, Tei K, Totsuka Y, Takinami $\mathrm{S}$ et al. Assessment of cervical lymph node metastasis using FDG-PET in patients with head and neck cancer. Am Nucl Med 2008;22(3):177-84.

7. Jeong HS, Baek CH, Son YI, Ki Chung M, Kyung Lee D, Young Choi $\mathrm{J}$ et al. Use of integrated (18)F-FDG PET/CT to improve the accuracy of initial cervical nodal evaluation in patients with head and neck squamous cell carcinoma. Head Neck 2007;29(3):203-10.

8. Kotani J, Kawabe J, Higashiyama S, Kawamura E, Oe A, Hayashi T et al. Evaluation of diagnostic abilities of Ga-SPECT for head and neck lesions. Ann Nucl Med 2008;22(4):297-300.

9. Le Bihan D, Breton E, Lallemand D, Aubin ML, Vignaud J, Laval-Jeantet M. Separation of diffusion and perfusion in intravoxel incoherent motion MR imaging. Radiology 1988; 168 (2): 497-505.

10. Padhani AR, Liu G, Koh DM, Chenevert TL, Thoeny HC, Takahara T, et al.. Diffusion-weighted magnetic resonance imaging as a cancer biomarker: consensus and recommendations. Neoplasia 2009 ; 11: 102-25.

11. Thoeny HC, De Keyzer F, King AD. Diffusionweighted MR imaging in the head and neck. Radiology. 2012 Apr;263(1):19-32.

12. Johnson JT. A surgeon looks at cervical lymph nodes. Radiology. 1990 Jun;175(3):607-10.

13. Rekha R, Reddy MVV, Reddy PP. Epidemiological Studies of Head and Neck Cancer in South Indian Population. Research In Cancer and Tumor 2013;2(2):38-44.

14. De Bondt RB, Hoeberigs MC, Nelemans PJ, Deserno WM, Peutz-Kootstra C, Kremer B et al. Diagnostic accuracy and additional value of diffusion-weighted imaging for discrimination of malignant cervical lymph nodes in head and neck squamous cell carcinoma. Neuroradiology. 2009;51 (3):183-92.

15. van den Brekel MW, Stel HV, Castelijns JA, Nauta JJ, van der Waal I et al. Cervical lymph node metastasis: assessment of radiologic criteria. Radiology. 1990;177(2):379-84. 
16. Takes RP, Righi P, Meeuwis CA, Manni JJ, Knegt P, Marres HA et al.. The value of ultrasound with ultrasound-guided fine-needle aspiration biopsy compared to computed tomography in the detection of regional metastases in the clinically negative neck. Int J Radiat Oncol Biol Phys 1998; 40 (5):1027-32.

17. Sumi M, Van Cauteren M, Nakamura T. MR microimaging of benign and malignant nodes in the neck. AJR Am J Roentgenol. 2006;186(3):749-57.

18. Ding ZX, Liang BL, Shen J, Xie BK, Huang SQ, Zhang B. Magnetic resonance imaging diagnosis of cervical lymph node metastasis from lingual squamous cell carcinoma. 2005;24(2): 199-203.

19. Sumi M, Sakihama N, Sumi T, Morikawa M, Uetani M, Kabasawa $\mathrm{H}$ et al. Discrimination of metastatic cervical lymph nodes with diffusionweighted MR imaging in patients with head and neck cancer. AJNR Am J Neuroradiol. 2003;24: 1627-1634.

20. Zhong J, Lu Z, Xu L, Dong L, Qiao H, Hua R et al. The Diagnostic Value of Cervical Lymph Node Metastasis in Head and Neck Squamous Carcinoma by Using Diffusion-Weighted Magnetic Resonance Imaging and Computed Tomography Perfusion. Biomed Res Int. 2014;2014:260859.
Original Research Article

21. Singh P, Parihar H, Goel V. Differentiating Benign and Malignant Metastatic Cervical Lymph Nodes by Diffusion Weighted MRI Sequence, International Journal of Anatomy, Radiology and Surgery, 2015;4(4):47-50.

22. ElSaid NAE, Nada OMMN, Semesim ARS, Habib YSEH, Semeisem AR et al. Diagnostic Accuracy of diffusion weighted MRI in diagnosing cervical lymphadenopathy correlated with pathology results. The Egyptian Journal of Radiology and Nuclear Medicine 2014; 45(4): 1115-25.

23. Perrone A, Guerrisi P, Izzo L, D’Angeli I, Sassi $\mathrm{S}$, Mele LL, et al. Diffusion-weighted MRI in cervical lymph nodes: differentiation between benign and malignant lesions. Eur J Radiol. 2011; 77 (2):281-6.

24. Wang J, Takashima S, Takayama F, Kawakami S, Saito A, Matsushita T. Head and neck lesions: characterization with diffusion weighted echoplanar MR imaging. Radiology. 2001; 220 (3): 621-30.

25. Abish YG, Roshdy HM, Enaite AM. Benign versus malignant cervical lns: differentiation by diffusion weighted MRI. AAMJ 2011;9(3):1-20.

\section{How to cite this article?}

Mathur M, Duhan V, Gupta R.K, Gupta S, Kaur N, Mathur A. The diagnostic accuracy of diffusion weighted magnetic resonance (MR) imaging for discrimination of malignant from benign cervical lymphadenopathy in head and neck tumours using histopathology as the reference standard. Int J Med Res Rev 2017;5 (12):10041014.doi:10.17511/ijmrr. 2017.i12.06 